\title{
EDMs in SUSY GUTs
}

\author{
Junji Hisano $^{\text {a }}$ \\ aICRR, University of Tokyo, 5-1-5 Kashiwa-no-Ha Kashiwa City, 277-8582, Japan \\ Hadronic and leptonic EDMs in SUSY GUTs are reviewed in this article.
}

\section{Introduction}

SUSY GUTs may predict rich flavor violation, and the signature may be observable in the low-energy flavor physics. The SUSY breaking terms in the minimal supersymmetric standard model (MSSM) are sensitive to physics beyond the MSSM. When the origin of the SUSY breaking terms in the MSSM comes from dynamics around or above the GUT scale, the interactions in the SUSY GUTs may bring down the signature on the SUSY breaking terms.

In the MSSM the sizable flavor-violating SUSY breaking (FVSB) terms for the left-handed squarks are induced by the large top-quark Yukawa coupling, and those for the left-handed sleptons may be also generated by the neutrino Yukawa interaction in the SUSY seesaw mechanism. In the SUSY GUTs the FVSB terms for those $\mathrm{SU}(5)$ partners, the right-handed squarks and sleptons, are generated. This gives a chance to probe the interactions at the GUT scale by the low-energy flavor-changing processes, such as the $K^{0}-\bar{K}^{0}$ mixing, the $B$ physics, and the lepton flavor violation.

The hadronic and leptonic electric dipole moments (EDMs) are sensitive to beyond the SM since the SM contributions are suppressed. In the MSSM the one-loop diagrams of SUSY particles may contribute to them. The EDMs are flavor-conserving observables, however, they are sensitive to the FVSB in the internal lines of the loop diagrams. Especially, when both left-handed and right-handed squarks or sleptons have the FVSB terms with the CP phases, the EDMs are enhanced by the heavier fermion mass. Thus, the EDMs are good probes for the SUSY GUTs.
In this paper we review the prediction of the hadronic and leptonic EDMs in the SUSY GUTs, and the implication to the other observables in the models.

\section{Hadronic EDMs}

When partons in nucleon have $\mathrm{CP}$ violating interactions, they contribute to the hadronic EDMs, such as ${ }^{199} \mathrm{Hg}$ and neutron EDMs. The current experimental bounds on neutron and ${ }^{199} \mathrm{Hg}$ atom EDMs are $\left|d_{n}\right|<6.3 \times 10^{-26} \mathrm{ecm}$ and $\left|d_{\mathrm{Hg}}\right|<1.9 \times 10^{-28}$ e cm, respectively (90\%C.L.) 11. The $\mathrm{CP}$ violation in the strong interaction of the light quarks is dictated by the QCD theta term and the quark CEDMs, $d_{q}^{C}(q=u, d, s)$, up to the dimension five terms. When the PecceiQuinn symmetry is imposed, the QCD theta parameter is suppressed.

The neutron and ${ }^{199} \mathrm{Hg}$ atom EDMs are evaluated as $d_{\mathrm{Hg}}=-8.7 \times 10^{-3} \times e\left(d_{u}^{C}-d_{d}^{C}+0.005 d_{s}^{C}\right)$ and $d_{n}=-1.6 \times e\left(d_{u}^{C}+0.81 \times d_{d}^{C}+0.16 \times d_{s}^{C}\right)[2]$. The contributions from the strange quark CEDM are included. From the SU(3) octet baryon mass splitting and the sigma term in the $N-\pi$ scattering, it is found that the strange quark component in nucleon is not negligible compared with other light quarks 3 .

Since the ${ }^{199} \mathrm{Hg}$ atom is diamagnetic, the EDM is sensitive to CP-violating nuclear force induced by the meson exchange, which generates T-odd EM potential parametrized by the Shiff moment. The latest evaluation of the Shiff moment [4] reveals that the the Shiff moment is sensitive to the isovector channel of the meson exchange. Thus, the strange quark CEDM contribution is suppressed by $\pi^{0}-\eta^{0}$ mixing. On the other hand, 
the neutron EDM is generated by nucleon-meson one-loop diagrams. The strange quark CEDM contribution to the neutron EDM is sizable compared with other light quarks. Here, the local counter term contribution to the neutron EDM is not included since it is not constrained by other observables. The theoretical evaluation still has large theoretical uncertainties.

From the experimental bound on the neutron $\left({ }^{199} \mathrm{Hg}\right) \mathrm{EDM}$, the CEDMs are bounded as $e\left|d_{u}^{C}\right|<3.9(2.2) \times 10^{-26}$ e cm, e $\left|d_{d}^{C}\right|<4.8(2.2) \times$ $10^{-26} \mathrm{e} \mathrm{cm}$, and $e\left|d_{s}^{C}\right|<2.4(44) \times 10^{-25} \mathrm{e} \mathrm{cm}$, assuming that the accidental cancellation among the CEDMs does not suppress the EDMs. Now the measurement of the deuteron EDM using the storage ring is proposed, and it is argued that the sensitivity may reach $d_{D} \sim 10^{-27}$ e cm [5]. This corresponds to $d_{u}^{C} \sim d_{d}^{C} \sim 10^{-28}$ e $\mathrm{cm}$ and $d_{u}^{C} \sim d_{d}^{C} \sim 10^{-26}$ e $\mathrm{cm}$ [2]. Thus, the further improvement may be possible.

As mentioned in Introduction, the hadronic EDMs are predicted by the FVSB in the SUSY GUTs. While the neutrino Yukawa coupling generates the right-handed sdown mixings, the FVSB induced by the top quark Yukawa coupling in cooperation of the CKM mixing is expected to dominate in the left-handed sdown mixings. Thus, we may probe the neutrino Yukawa coupling by using the hadronic EDMs 6 .

In Fig. 1 the down and strange quark CEDMs in the SUSY SU(5) GUT with the right-handed neutrinos are presented [6]. The right-handed sdown mixing between the second and third generations, depend on $U_{\mu 3}$ with $U$ the MNS matrix for the neutrino mixing, generates $d_{s}^{C}$. It is found from this figure that the right-handed tau neutrino mass larger than about $10^{14} \mathrm{GeV}$ are excluded from the bound on $d_{s}^{C}$. On the other hand, $d_{d}^{C}$ is sensitive to $U_{e 3}$ via the correction to the the right-handed sdown mixing between the first and third generations. At present the constraint from $d_{d}^{C}$ is weak. However, the further improvement of the hadronic EDM measurements, such as the deuteron EDM, may give a significant impact.

Now we discuss the implication of the hadronic EDM constraints to other phenomena. The Belle and BaBar experiments reported recently that the $\mathrm{CP}$ asymmetries in the $b-s$ penguin processes
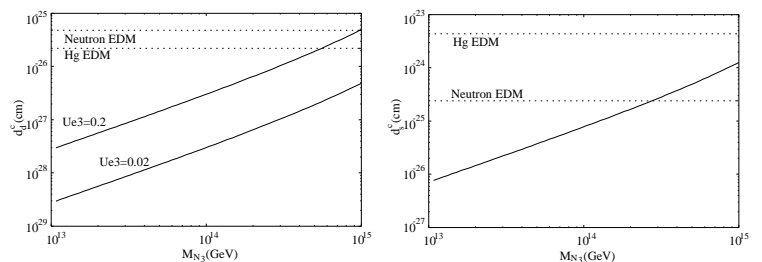

Figure 1. CEDMs for the strange quark in (a) and for the down quark in (b) as functions of the right-handed tau neutrino mass, $M_{N_{3}}$. Here, $U$ is the MNS matrix. See Ref. [6] for the input parameters.

in the $B$ decay, including $B \rightarrow \phi K_{s}$, are deviated from the SM prediction [7]. The $b-s$ penguin is the radiative process even in the $\mathrm{SM}$, and it is sensitive to beyond the SM. It is pointed out that the right-handed sdown mixing between the second and third generations, which is predicted in the SUSY GUTs, may give sizable corrections to the processes [8]. However, the constraint on the right-handed sdown mixing from the hadronic EDMs implies that the deviation in the $b-s$ penguin processes in the SUSY GUTs should be suppressed 92. In Fig. 2] we show the correlation between $d_{s}^{C}$ and the CP asymmetry in $B \rightarrow \phi K_{s}$ $\left(S_{\phi K_{s}}\right)$ assuming the non-vanishing right-handed sdown mixing. From this figure, it is found that the deviation of $S_{\phi K_{s}}$ from the SM prediction $(0.731 \pm 0.056)$ is suppressed. In addition to this correlation, the EDMs and $\operatorname{Br}(\tau \rightarrow \mu \gamma)$ are also correlated with each other due to the GUT relation among the SUSY breaking terms [106]. The constraint on $d_{s}^{C}$ implies $B r(\tau \rightarrow \mu \gamma) \lesssim 10^{-(7-8)}$.

\section{Leptonic EDMs}

The leptonic EDMs, such as muon and electron EDMs, are also predicted in the SUSY GUTs. Even in the minimal SUSY seesaw model the EDMs are generated by the threshold correction to the FVSB terms, however, they are smaller [11. In the SUSY GUTs, the left-handed slep- 


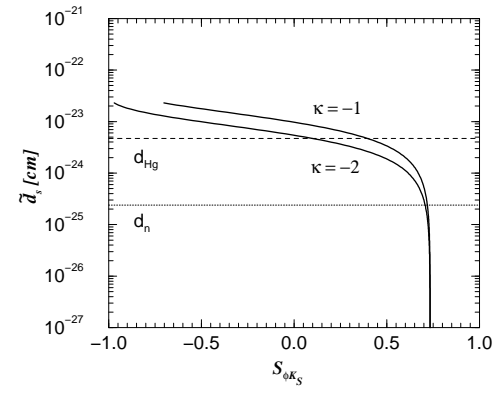

Figure 2. The correlation between $d_{s}^{C}$ and the $\mathrm{CP}$ asymmetry in $B \rightarrow \phi K_{s}\left(S_{\phi K_{s}}\right)$. The dashed (dotted) line is the upperbound on $d_{s}^{C}$ from the EDM of ${ }^{199} \mathrm{Hg}$ atom (neutron). See Ref. [2] for the input parameters.

ton mixings are induced by the neutrino mixing while those for the right-handed sleptons are parameterized by the CKM mixing at the GUT scale. The leptonic EDMs are correlated with $\operatorname{Br}(\mu \rightarrow e \gamma)$, and null result in the $\mu \rightarrow e \gamma$ search gives bounds on the leptonic EDMs.

The effective operator for $\mu \rightarrow e \gamma$ is $H=$ $\bar{e}(F \sigma)\left(A_{R} P_{L}+A_{L} P_{R}\right) \mu$. While $A_{L}$ has the various contributions, the dominant contribution to $A_{R}$ comes from a diagram proportional to $m_{\tau}$. The electron (muon) EDM is also generated by a similar diagram proportional to $m_{\tau}$. Since $A_{L}$ and $A_{R}$ are not interfered with each other, the experimental bound, $\operatorname{Br}(\mu \rightarrow e \gamma)<$ $1.2 \times 10^{-11}$, gives the upperbounds on the leptonic EDMs as $\left|d_{e}\right| \lesssim 3 \times 10^{-26}\left|U_{e 3} / U_{\mu 3}\right|$ e cm and $\left|d_{\mu}\right| \lesssim 3 \times 10^{-26}\left|V_{32} / V_{31}\right| e \mathrm{~cm}$. Here, $V$ is the CKM matrix at the GUT scale. Thus, $\left|d_{e}\right| \lesssim 10^{-(26-27)}$ e $\mathrm{cm}$, and this is comparable to the current experimental bound. For the muon EDM, $\left|V_{32} / V_{31}\right| \sim 5$ implies $\left|d_{\mu}\right| \lesssim 10^{-25} \mathrm{e} \mathrm{cm}$. If $V_{31}$ is much smaller than the measured value at low energy and $\operatorname{Br}(\mu \rightarrow e \gamma)$ is more suppressed, $V_{32} \sim 0.04$ leads to $\left|d_{\mu}\right| \lesssim 10^{-24}$ e $\mathrm{cm}[10$.

\section{Summary}

In this article, the hadronic and leptonic EDMs in the SUSY GUTs are reviewed. The EDMs are sensitive to the FVSB for squarks and sleptons generated by the GUT scale interaction. The EDMs are traditional, however, still stringent constraints on the model beyond the SM, and it is important to take correlations of the EDMs with other low energy observables.

\section{REFERENCES}

1. P. G. Harris et al., Phys. Rev. Lett. 82 (1999) 904; M. V. Romalis, W. C. Griffith and E. N. Fortson, Phys. Rev. Lett. 86 (2001) 2505.

2. J. Hisano and Y. Shimizu, Phys. Rev. D 70 (2004) 093001.

3. A. R. Zhitnitsky, Phys. Rev. D 55 (1997) 3006; V. M. Khatsimovsky, I. B. Khriplovich and A. R. Zhitnitsky, Z. Phys. C 36 (1987) 455 .

4. V. F. Dmitriev and R. A. Sen'kov, Phys. Atom. Nucl. 66 (2003) 1940 [Yad. Fiz. 66 (2003) 1988].

5. Y. K. Semertzidis et al. [EDM Collaboration], AIP Conf. Proc. 698 (2004) 200.

6. J. Hisano, M. Kakizaki, M. Nagai and Y. Shimizu, hep-ph/0407169

7. Talked by Y. Sakai and M. Giorgi in 32nd International Conference On High-Energy Physics (ICHEP 04) 16-22 Aug 2004, Beijing, China.

8. T. Moroi, Phys. Lett. B 493 (2000) 366.

9. J. Hisano and Y. Shimizu, Phys. Lett. B 581 (2004) 224.

10. J. Hisano and Y. Shimizu, Phys. Lett. B 565, 183 (2003).

11. J. R. Ellis, J. Hisano, M. Raidal and Y. Shimizu, Phys. Lett. B 528 (2002) 86; Y. Farzan and M. E. Peskin, arXiv:hep-ph/0405214. 